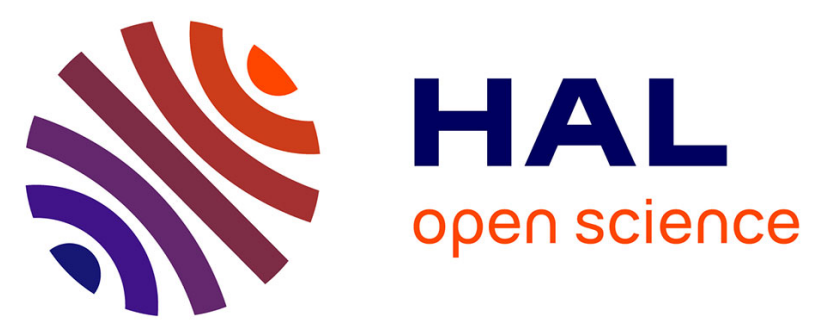

\title{
Patterned neuronal networks using nanodiamonds and the effect of varying nanodiamond properties on neuronal adhesion and outgrowth
}

\author{
R. Edgington, A. Thalhammer, J. O. Welch, A. Bongrain, P. Bergonzo, \\ Emmanuel Scorsone, R. Jackman, R. Schoepfer
}

\section{To cite this version:}

R. Edgington, A. Thalhammer, J. O. Welch, A. Bongrain, P. Bergonzo, et al.. Patterned neuronal networks using nanodiamonds and the effect of varying nanodiamond properties on neuronal adhesion and outgrowth. Journal of Neural Engineering, 2013, 10, pp.056022. 10.1088/1741-2560/10/5/056022 . cea-01791988

\section{HAL Id: cea-01791988 https://hal-cea.archives-ouvertes.fr/cea-01791988}

Submitted on 18 Feb 2019

HAL is a multi-disciplinary open access archive for the deposit and dissemination of scientific research documents, whether they are published or not. The documents may come from teaching and research institutions in France or abroad, or from public or private research centers.
L'archive ouverte pluridisciplinaire HAL, est destinée au dépôt et à la diffusion de documents scientifiques de niveau recherche, publiés ou non, émanant des établissements d'enseignement et de recherche français ou étrangers, des laboratoires publics ou privés. 
Patterned neuronal networks using nanodiamonds and the effect of varying nanodiamond properties on neuronal adhesion and outgrowth

Robert Edgington ${ }^{\text {a1 }}$, Agnes Thalhammerb1, Joseph Welcha, Alexandre Bongrain $^{c}$, Philippe Bergonzoc, Emmanuel Scorsonec ${ }^{c}$, Richard B. Jackmana, Ralf Schoepferb

a London Centre for Nanotechnology and Department of Electronic and Electrical Engineering, University College London, 17-19 Gordon Street, London, WC1H 0AH, UK

b Laboratory for Molecular Pharmacology, NPP, University College London, Gower Street, London, WC1E 6BT, UK

c CEA, LIST, Diamond Sensors Laboratory, F-91191 Gif-sur-Yette, France

* corresponding author r.edgington@ucl.ac.uk +44(0)2076799931

1 These authors contributed equally

\section{$\underline{\text { Abstract }}$}

Detonation nanodiamond monolayer coatings are exceptionally biocompatible substrates for in-vitro cell culture. However, the ability of nanodiamond coatings of different origin, size, surface chemistry and morphology to promote neuronal adhesion has yet to be investigated. Herein, the ability of various nanodiamond coatings of different type to promote neuronal adhesion is investigated and the resulting universal promotion of neuronal adhesion on all nanodiamond coatings is discussed with respect to coating parameters to infer the mechanisms behind neuronal attachment and subsequent outgrowth. Furthermore, neuronal patterning on artificial substrates is achieved using patterned nanodiamond coatings down to at least $10 \mu \mathrm{m}$, which shows great promise for applications both in-vitro and in-vivo. 


\section{Introduction}

Nanodiamond (ND) coatings are extremely biocompatible substrates for interfacing with living cells and are excellent platforms for supporting even the most environmentally demanding cell types $[1,2]$ using minimal culture conditions. In particular, detonation nanodiamond (DND) monolayers have been shown [2] to support cultures of hippocampal neurons - arguably one of the most environmentally demanding cell types. In this case nanodiamond monolayers promote the attachment and formation of functional neuronal networks even in the absence of the otherwise prerequisite procedure of laminating culture surfaces with adhesion promoting extracellular matrix (ECM) proteins (e.g. laminin) prior to cell seeding. Moreover, DND monolayers show a remarkable ability to support neuronal networks in stark comparison to the similar material of nanocrystalline diamond (NCD) thin films [2]. Considering the difference in ability of DNDs and NCD to support neuronal cultures, yet the seemingly similar surface properties of these materials, the mechanism of neuronal attachment onto DND monolayers remains to be addressed. The proteomic dimensions, chemical inertness and organic surface functionalisations of monodispersed nanodiamonds and their coatings suggest DNDs to have an influence on neuronal adhesion on a nanoscale subcellular level. Therefore in this study the size, surface chemistry, source and deposition method of nanodiamond coatings have been varied and parametrised in order to investigate their influence on neuronal adhesion and gain insight into the mechanism of neuronal adhesion observed on ND-coated substrates. Furthermore, in view of the promising nature of ND monolayers for in vitro and in vivo brain-machine interface application due to their ease of deposition in 3D on most materials [3], porous nature and electrode functionality [4], the usage of micropatterning for the directed growth of hippocampal neurons is demonstrated using patterned DND coatings of varying line widths from 5 to $70 \mu \mathrm{m}$. 
$\underline{\text { Experimental Methods }}$

\section{$\underline{\text { Chemicals }}$}

All chemicals were from Sigma (St Louis, MO), Tocris (Tocris Cookson Ltd, Avonmouth, UK) or Invitrogen (Carlsbad, CA), unless stated otherwise. For all experiments substrates of borosilicate cover slip glass were used Glass (13 Ø cover glass, VWR, UK). Samples were sterilized by autoclave following ND deposition, which did not affect ND surface functionalisation, as determined by Fourier Transform Infrared Spectroscopy (FTIR) (data not shown). Various ND types and deposition methods were used as follows.

ND types: Monodispersed DNDs (mDND, 6-10 nm) were purchased from New Metals \& Chemicals Corporation, Japan, and larger, purposefully aggregated (aDND, ca. 6-20 nm) DND hydrocolloids from the same source were prepared by Dr Oliver A Williams (Fraunhofer Institute for Applied Solid State Physics, Freiburg, Germany). High Pressure High Temperature (HPHT) Syndia SYP 0-0.02 GAF aqueous solution nanodiamonds were purchased from Van Moppes (ca. $20 \mathrm{~nm}$ ). Deposition methods: Ultrasonication (US): Samples were submerged in 0.5 g/L ND hydrocolloid (mDND used) and subjected to $10 \mathrm{mins}$ ultrasonication, then dried by $\mathrm{N}_{2}$ gun. Spin coating (SC): $0.5 \mathrm{~g} / \mathrm{L}$ ND hydrocolloids (mDND and aDND used) were coated on whole substrates, then spun at $2000 \mathrm{rpm}$ for 20 seconds and dried by $\mathrm{N}_{2}$ gun. PVA/PDDAC electrostatic coating: NDs (aDND used) were deposited using electrostatic attraction as described previously [5], where negatively charged NDs electrostatically bind to positively charged polydiallyldimethylammonium chloride (PDDAC). Remaining solvent and polymers were removed by a short (20-40s) hydrogen microwave plasma using a Seki AsteX AX6550 reactor and the following parameters: MW power, $600 \mathrm{~W} ; \mathrm{H}_{2}, 200 \mathrm{sccm}$; pressure, 20 mbar in order to keep the measured temperature to $500^{\circ} \mathrm{C}$ (as 
probed using an optical pyrometer Ircon Modline 3 series 3L). Dip: Samples were coated in PDDAC and dipped in $3 \mathrm{~g} / \mathrm{L}$ HPHT NDs. PDDAC was removed using the same hydrogen plasma as above.

Following coating, some samples were treated to a short microwave plasma enhanced chemical vapour deposition (MWPECVD) process that served to fixate the NDs on the surface, also resulting in a hydrogenated diamond surface. The parameters used were as above for the PVA/PDDAC removal, except with a $1 \%$ methane addition and a run time of 11 minutes.

\section{Patterning}

Patterning of NDs was achieved as described previously [6] using a photolithography mask comprised of a filled surface (control), a grid of constant pitch $(200 \mu \mathrm{m})$ and gradated line widths of 5, 10, 15, 20, 25, 30, 35, 40, 50, 60 and $70 \mu \mathrm{m}$ (Figure 3a). In brief, following ND coating an Al hard mask was deposited and patterned using AZ NLOF 2020 photoresist and AZ351B developer, which simultaneously developed the resist and etched the $\mathrm{Al}$ hard mask. The exposed NDs were then etched away using an $\mathrm{O}_{2} / \mathrm{Ar}$ reactive ion etch and the remaining resist and $\mathrm{Al}$ removed, followed by multiple deionized water rinses.

\section{$\underline{\mathrm{AFM}}$}

AFM measurements were carried out using a NanoScope V (7.0) controlled Dimension V Scanning Probe Microscope equipped with an XYZ Hybrid Scanner (Veeco Instruments Inc., Mannheim, Germany), using aluminium-coated silicon AFM probes with a resonant frequency of 190 $\mathrm{kHz}$ (Nanoscience Instruments, Inc., Phoenix, USA). The AFM was operated in tapping mode with a VT-103-3K Acoustic/ Vibration Isolation System and the VT-102 Vibration Isolation Table at room temperature in air. Statistical analysis and image processing on AFM images was carried out using Gwyddion software. Firstly topographical data was tilt corrected using a first order plane fit subtraction followed by scar removal and median line correction where necessary. Average roughness, $R_{a}$, was 
calculated using the following formula $\frac{1}{\square} \sum_{j=1}^{\square}\left|Z_{j}\right|$ where $\mathrm{N}$ is the number of data points and $\mathrm{Z}$ is the topographical data (hereafter $\mathrm{N}$ refers to seeding density of NDs). Particle analysis was then performed on all images. Firstly a low band pass 1D FFT filter was applied to all images to filter out high frequency noise (Fourier modulus density of $>0.2 \mathrm{k} \mathrm{nm}^{-1}$ removed). Particle marking was performed using a semiautomatic process of either a watershed or threshold detection method followed by manual editing of the particle masks. Falsely marked particles of insufficient size were removed using a threshold area filter. Particle statistics of seeding density $(\mathrm{N})$ and equivalent disc radius (EDR), which is the radius of a disc of the same area as the marked grain, were then calculated. The standard error of the mean EDR was then calculated to represent the breadth of each EDR distribution for each layer. It should be noted EDR does not truly reflect particle size due to AFM tip convolution effects. Tip convolution resulted in overestimates of EDR by ca. $10 \mathrm{~nm}$, as estimated from the $\mathrm{x}$-axis intercept of the $\mathrm{z}$-axis height of grains vs. their EDR.

\section{$\underline{\text { Raman }}$}

Raman spectroscopy was carried out with a Renishaw Invia Raman Spectrometer using a laser wavelength of $514.5 \mathrm{~nm}$. Thicker ND coatings were used for characterisation, prepared by evaporating ND hydrocolloid drops on Si wafers in order to be visible under the Raman Spectrometer.

\section{Primary neuronal cultures}

Murine hippocampal neurons were obtained at embryonic day 18, essentially as described in [7]. For cells cultured on ECM proteins, glass coverslips were incubated overnight in $100 \mu \mathrm{L}$ of a $3 \mu \mathrm{g} / \mathrm{mL}$ poly-ornithine (p-ORN) plus $2 \mu \mathrm{g} / \mathrm{mL}$ laminin (LN) solution in PBS. Cells were seeded in attachment medium (AM; MEM with Earl's salts and glutamine, 10\% fetal bovine serum (FBS), $33 \mathrm{mM}$ glucose, $1 \mathrm{mM}$ pyruvate) at 100,000 cells/cm². After 3-4 h the AM was replaced with maintenance medium (MM; Neurobasal medium, 2\% B27 supplement, 2 mM GlutaMAX-I, 100 U/ml 
penicillin, $0.1 \mathrm{mg} / \mathrm{ml}$ streptomycin, $33 \mathrm{mM}$ glucose). Cultures were kept for up to 7 days in vitro (DIV7). All animal work was undertaken under the auspices of the UK Home Office Project and Personal Licenses held by the authors in their designated laboratories.

\section{Immunochemistry and imaging}

Samples were fixed with $4 \%$ paraformaldehyde/4\% sucrose in phosphate buffered saline (PBS; $137 \mathrm{mM} \mathrm{NaCl}, 2.7 \mathrm{mM} \mathrm{KCl}, 10 \mathrm{mM} \mathrm{Na}_{2} \mathrm{HPO}_{4}, 2 \mathrm{mM}$ $\mathrm{KH}_{2} \mathrm{PO}_{4}$ ) for 10 min, washed with PBS before permeabilisation of cells with $0.2 \%$ TritonX-100 in PBS for 10 min. After $1-2 \mathrm{~h}$ in blocking solution (BS; 5\% horse serum in PBS) samples were incubated for 1-2 $\mathrm{h}$ with primary antibodies (mouse anti-MAP2, Chemicon, MAB364, 1:500 in BS; rabbit anti-GFAP, DakoCytomation, Z0334, 1:500 in BS). F-actin was stained for $10 \mathrm{~min}$ at RT with $2 \mathrm{U} / \mathrm{ml}$ rhodamine-phalloidin (Molecular Probes R415) and nuclei for 5 min at RT with Hoechst 33258 (Molecular Probes, $1 \mathrm{mg} / \mathrm{ml}$ in PBS). Samples were washed repeatedly and incubated with secondary antibody (Cy2- and $\mathrm{Cy} 5$-conjugated goat anti-mouse IgG, Jackson; 1:300 in BS, 115-095-003 and 115-175-003, respectively) for $1 \mathrm{~h}$. Samples were washed with PBS, and stored at $4{ }^{\circ} \mathrm{C}$ in sterile-filtered PBS until imaging or mounted after wash in Gelmount (Sigma).

Confocal microscopy was performed on a Bio-Rad Radiance 2100MP Confocal Microscope with a 20x (Nikon, Plan Flour, 0.75) water-immersion objective using Bio-Rad Lasersharp 2000 software. Alternatively a Leica TCS SPE confocal microscope equipped with a 40x oil-immersion objective (Zeiss, ACS APO, 1.15) was used in combination with LAS AF software, V1.7.0. Areas for imaging were randomly chosen. 2 independent sets of experiments were performed for unpatterned cultures, and 1 independent set for patterned cultures. Total neurite length (TNL) measurements were performed using a modified version of the NeuriteTracer ImageJ plugin [8] that does not require nuclei markers. TNL was calculated on one set of confocal images of unpatterned DIV2 neuronal cultures. Image processing was performed on the neuronal marker confocal image channel, which had 
been recorded using the same confocal microscope settings for each image. Processing thresholds were chosen individually for each image to most faithfully trace the neurites. TNL was measured as an average of 4 quadrants of each confocal image in order to reflect the heterogeneity of neuronal cultures, and its associated error is presented as the standard error of the mean. Each quadrant has a surface area of $98,775 \mu \mathrm{m}^{2}$. Pearson product-moment correlation coefficients of TNL with respect to ND coating morphology parameters were calculated using OriginPro 8.6 software, where p-values were calculated using an unpaired Student's 2tailed t-test (significant for $\mathrm{p}<0.05$ ).

\section{$\underline{\text { Results }}$}

\section{ND coating and characterisation}

To investigate the dependence of ND coating type on neuronal adhesion, ten different types of coatings have been prepared on glass slides using different ND sources, deposition methods, and with or without the addition of a chemically modifying PECVD treatment. Firstly, monodispersed DNDs (mDNDs) were deposited on glass cover slips using ultrasonic seeding (US) as reported previously [2] (Figure 1a 1 $1^{\text {st }}$ column). The same mDNDs were next deposited by spin coating (SC), resulting in more aggregated, sparse DND coatings (Figure 1a, $2^{\text {nd }}$ column, rows $1 \&$ 2). In order to investigate the effect of ND particle size on neuronal adhesion, aggregated DND (aDND) hydrocolloids were then prepared, deposited via the methods of spin coating (Figure 1a, $3^{\text {rd }}$ column, rows $1 \&$ 2) and electrostatic binding using ND/PVA hydrocolloids on PDDACcoated glass substrates (hereafter referred to as 'PVA'; see Experimental Methods) (Figure 1a, $4^{\text {th }}$ column, rows $1 \& 2$ ). US seeding was not used for aDNDs due to it possibly effecting aggregate size. Finally, NDs synthesised by the HPHT method were used in order to investigate ND coatings of different origin. HPHT NDs have a similar but differing 
surface chemistry, size (ca. $20 \mathrm{~nm}$ ) and morphology to NDs of detonation origin.

For each coating type above additional duplicate samples were then prepared which, following deposition, were subjected to a short MWPECVD treatment in order to result in better-adhered ND coatings and also hydrogenated ND surfaces, as opposed to untreated, predominantly oxygenated ND surface functionalisation [9]. Following MWPECVD treatment, the increased adhesion of ND coatings was observed via the improved resistance of the coatings to mechanical abrasion. Furthermore the improved adhesion of ND layers was demonstrated by the elimination of unwanted AFM measurement phenomena associated with measuring loose nanopowders, such as inconsistent feature scanning from particles being moved around by the tip and tip artefacts (anomalous reoccurring shapes convoluted into the AFM image) from NDs becoming stuck onto the tip. MWPECVD processing is commonly used to grow nanocrystalline diamond (NCD) films [10] from ND seeded coatings, therefore, in order to not result in an accrued mass of diamond on the NDs nor significantly alter their morphology, the fixing step was kept short (11 mins) and at relatively low power in comparison to typical diamond growth parameters. AFM image pairs of most coatings (Figure 1a, 1b) revealed no discernable differences in the morphology of the coatings before and after fixation outside of the heterogeneity expected from the employed deposition methods, except for perhaps the sample 'fixated aDND PVA', which had a skewed average roughness $\left(R_{\mathrm{a}}\right)$ measurement that could be ascribed to an atypical coating heterogeneity for the fixated sample area measured. The average roughness $\left(\mathrm{R}_{\mathrm{a}}\right)$ and ND particle statistics of equivalent disc radius (EDR) and seeding density $(\mathrm{N})$ were measured from AFM images as described in Experimental Methods, and parametrised ND coating properties are displayed in Figure 1b. 
In order to confirm the lack of increase in ND particle size after MWPECVD fixation treatment, Raman spectroscopy was performed on thicker (ca. $100 \mathrm{~nm}$ ) mDND layers before and after treatment. All samples were not tested due to the smallest diamond seeds being the most sensitive to changes in Raman spectra with respect to increased particle size (see below). Raman spectra in Figure 1c show the crystalline carbonaceous structure of the fixated mDND to be very similar to that of untreated nanodiamond coatings and distinct from grown NCD films [11]. Pertinently, the redshift of the $\mathrm{sp}^{3}$ diamond peak from $1333 \mathrm{~cm}^{-1}$ to 1327 $\mathrm{cm}^{-1}$ observed in both Raman spectra indicates the primary particle size of the mDNDs to remain unaltered by the MWPECVD fixation (the redshift arises from phonon confinement in nanodiamond and is related to particle size [12]).

\section{Neuronal cell attachment on ND coatings}

Primary neuronal cultures were seeded onto the ten differently ND-coated samples and control sample of a glass cover slip coated with LN/p-ORN. Confocal microscopy images of immunostained neuronal cultures after 2 days in vitro (DIV2) revealed attachment and outgrowth of neurons on all samples tested (Figure 2). In order to quantify neuronal outgrowth on the different ND coatings, the total neurite length (TNL) was measured as described in Experimental Methods. Average TNL per quadrant for all samples is shown in the bottom right hand panel of Figure 2. For NDcoated substrates neurite extension was similar or in excess to the control LN/p-ORN coated substrate. Notably, no discernable differences between untreated and hydrogenated fixated ND coatings were observed, except for samples untreated and fixated aDND SC that showed a reduced neurite extension post-fixation (Figure 2, $3^{\text {rd }}$ row).

Having parametrised the degree of neurite extension on the different NDcoated samples, the Pearson product-moment correlation coefficients, $r$, 
and their significance, s, were calculated in order to test for any significant linear correlation between TNL and the associated ND coating parameters of $R_{a}$, EDR and N. Scatter plots of $R_{a}$, EDR and N vs. TNL are shown in Figure 3, accompanied by their associated $r$ and s values inset in each panel. The largest and only statistically significant $(r=-0.77 \mathrm{p}=0.01)$ correlation observed is EDR vs. TNL, which suggests that the size of particles could have a determining factor on neuronal adhesion and development in the nanoscale range tested. $R_{a}$ also shows a negative but

insignificant correlation coefficient over the $R_{a}$ range tested. No significant correlation to TNL is observed for the $\mathrm{N}$ range tested, however the correlation coefficient for $\mathrm{N}$ vs. TNL is positive $(\mathrm{r}=0.4, \mathrm{p}=0.25)$ as expected (as $\mathrm{N}$ tends to zero so should TNL). Cultures were maintained up to at least DIV7, forming apparent neuronal networks on all substrates tested (data not shown).

\section{$\underline{\text { ND-directed neuronal patterning }}$}

Patterned ND coatings were deposited as described in Experimental Methods using the photolithography mask pictured in Figure 4a. The ND type and deposition method of untreated aDND SC was used due to its spin coating process being readily incorporated into photolithography processing and it also having a high seeding density for ease of feature identification using an AFM. ND patterns were etched with little residual ND particulates and Figure 4b shows an AFM image of a crossroad junction of $15 \mu \mathrm{m}$ thick ND tracks. Neuronal cultures were performed as above on top of patterned substrates. Confocal microscopy images after DIV2 reveal NDs to be able to directly pattern neuronal adhesion down to line widths of at least $10 \mu \mathrm{m}$ (Figure 4c). Upon inspection of Figure 4c.i one can see the majority of neuron nuclei sit over the junction of the ND tracks for $10 \mu \mathrm{m}$ line widths, whereas for larger widths (Figure 4c.ii, iii) nuclei are dispersed seemingly irrespective of the patterned features. For the unpatterned area of Figure 4c.iv, less neurite branching is observed 
than on the patterned areas, with the neurons taking on a more circular morphology, as also observed in aDND SC in Figure 2. After DIV7 (Figure 4c.v) neurons can be seen to have formed physically connected, ordered networks on top of the ND tracks and, in comparison to the unpatterned mask area (Figure 3c.vi), the neurons are not fasciculated.

\section{$\underline{\text { Discussion }}$}

\section{Mechanism of neuronal adhesion with respect to ND surface properties}

Whilst cellular adhesion is regularly achieved on artificial substrates via biomolecule and peptide lamination, some artificial substrates have been shown to be able to promote cellular adhesion. The most primitive mechanism known to promote cellular adhesion on artificial substrates is the electrostatic binding of negatively charged cell membranes to positively charged surfaces of materials such as polylysine [13]. NDs could also have a similar effect on neuronal adhesion especially considering their polar surfaces, however, a common fate for cells adhering in such manner is apoptosis that is caused by cells' subsequent inability to secrete ECM proteins [14]. NDs could be providing an electrostatically attractive substrate for initial cellular adhesion and moreover, due to their nanoscale porosity, also be providing a platform for endogenous ECM formation and continued cell survival. However, this is unlikely because neuronal growth is supported on both hydrogenated and oxygenated coatings (Figure 2) which possess opposite charges.

Considering the remarkable ability of NDs to support and direct neuronal adhesion for extended periods of time, it is tempting to entertain the possibility that NDs are mimicking the functional properties of ECM proteins and are directly interfacing with cells and initiating cellular attachment and development. Afterall, the organic functionality, size, charge and shape of ND particles is similar to that of globular proteins, 
and other nanoparticles have already been shown to mimic other biological processes [15]. In the particular case of NDs being anchors for the development of focal adhesions with neurons, NDs would have to mimic the function of adhesion molecules (AMs) of the ECM by mimicking AM peptide motifs such as (for laminin) RGD, YIGSR, IKVAV, etc. [16], which in turn interface with cells via membrane proteins called integrins [17]. In this context, similarities can be drawn between ND surface functionalities and the functionalities of R-groups of amino acids of AM motifs, such as the amine groups on Arginine (R) and carboxylic groups on Aspartic Acid (D) of the common RGD motif. Whilst it is not inconceivable that functionalities on NDs could be serendipitously arranged as to occasionally mimic AMs' motifs (especially considering organic RGD mimetics exist [18]), direct ND-cell interaction is still highly unlikely. Without integrin signalling cells die in a process called anoikis, and even slight changes in integrins or AM motif sequences cause anoikis or severe loss of function in cells $[19,20]$. Therefore, it would be surprising that NDs - crudely forged in detonation chambers - could mimic the highly specific, evolved interactions of AMs with integrins. In this context direct cell-ND interaction is refuted by the observation of neuronal adhesion on both untreated and hydrogenated NDs; the latter of which could not have such AM motif mimetic surface chemistry.

Instead, it is much more likely that NDs provide a substrate for the optimal adsorption of AMs, such as vitronectin and fibronectin from serum proteins used in the attachment stage of cell culturing and the subsequent ECM proteins produced by cells themselves [17], much in the same way that tissue culture polystyrene promotes cellular adhesion [21,22]. Indeed, in the absence of serum neurons do not attach to ND-coated substrates (n.b. serum also contains other growth factors essential to cell attachment that are not AMs) and the slight fasciculation of neurons observed in Figure 4c.v (after DIV7) can be ascribed to neurons attaching onto their own matrix of endogenous AMs, and not the underlying surface (n.b. 
fasciculation has also been observed previously [2] on control ECM coated substrates and therefore can also be ascribed to varying culture conditions).

From previous studies [2] it has been shown that nanocrystalline diamond (NCD) films, which are similar in surface functionalisation and bulk constitution to NDs but differ in surface morphological properties such as curvature, do not usually promote murine hippocampal neuronal growth. The remarkable attachment of neurons observed in [2] and herein on NDcoatings can most probably be explained by functional, well-orientated AM protein adsorption onto NDs as a result of their small radii of curvature; not simply an apt average nanoscale roughness, as well as their high affinity for protein adsorption [23] (n.b. NCD used in [2] was of similar roughness to ND coatings). The NDs used in this study all have radial curvatures in the order of that of proteins, resulting in point-contact adsorption that allows proteins to stay strongly electrostatically adhered to NDs but remain in their native structural and functional shape, as dictated by the hydrophobic force. For example, it has recently been shown that the bovine serum albumin (BSA) protein adsorbs with high affinity to NDs whilst retaining its structural features [24]. In this context, the correlation coefficient analysis in Figure 3 between the neurite extension and parametrised morphological measureables of the ND coatings show a significant correlation between the radii of curvature (proportional to EDR) of the ND particles and the total neurite length measured $(r=-0.77$, $\mathrm{p}=0.01$ ), whereas the more commonly studied biomaterial metric of average roughness is only weakly correlated to neurite extension ( $\mathrm{r}=-0.48$, $\mathrm{p}=0.16$ ). The negative correlation measured agrees with the above hypothesis that as the ND particles tend towards the size of proteins, the surface becomes more biocompatible and more neurite extension is observed. 
In consideration of which AMs could be responsible for initial attachment of neuronal cells to ND-coatings, vitronectin has been shown to absorb with high affinity to NDs from serum [23] and is known to be an important AM for initial cell attachment [17,22]. Furthermore, vitronectin is of similar size to BSA and is made up of globular domains that are easily denatured on low curvature surfaces during absorption [25,26], which could explain the increased adhesion of neurons on ND-coatings and reduced adhesion on facetted low curvature NCD.

\section{$\underline{\text { Conclusions }}$}

Various nanodiamond coatings of different origin, size, aggregation and deposition method have been investigated for their ability to support neuronal adhesion and all ND coatings are shown to universally promote murine hippocampal neuronal adhesion. In particular interest, predominantly oxygenated (untreated) and hydrogenated, fixated nanodiamond coatings show no apparent differences in their ability to promote neuronal adhesion. The possible mechanisms for neurons adhering to ND coatings are discussed, and via parametric correlation coefficient analysis of the morphological properties of ND coatings and their corresponding neuronal cultures, the small radii of curvature of NDs, and thereof their ability to adsorb ECM proteins in their functional state, is implicated as a determining factor in the ability of ND coatings to support neuronal adhesion and growth. Using photolithography and reactive ion etching, nanodiamond patterns are fabricated and the direct patterning of neuronal adhesion using nanodiamond is demonstrated, making ND patterning a promising technique for the in vitro study of ordered neuronal networks without using additional biomolecules to promote adhesion. Furthermore, patterned ND coatings show great potential for directing neurons towards electrodes of neuroprosthetics such as artificial retina microelectrode arrays or cochlear implants. 
$\underline{\text { Acknowledgements }}$

The work was financially supported by awards to RBJ and RS by the UK's Engineering and Physical Sciences Research Council (EPSRC, EP/F026110) and an EU Framework 6 STREP programme 'DREAMS' (FP6-NMP- 033345). Dr Oliver A. Williams (IAF, Freiburg, Germany now at University of Cardiff, UK) is thanked for his skillful preparation of the dispersed ND solutions that were used throughout. Dr Madeline Pool (McGill University, Canada) is thanked for her kind provision of the modified NeuriteTracer software. At UCL Dr Steve Hudziak is acknowledged for his assistance with the AFM measurements.

\section{$\underline{\text { References }}$}

[1] Lechleitner T, Klauser F, Seppi T, Lechner J, Jennings P, Perco P, Mayer B, Steinmueller-Nethl D, Preiner J, Hinterdorfer P, Hermann M, Bertel E, Pfaller K, Pfaller W. The surface properties of nanocrystalline diamond and nanoparticulate diamond powder and their suitability as cell growth support surfaces. Biomaterials 2008;29:4275-84.

[2] Thalhammer A, Edgington RJ, Cingolani LA, Schoepfer R, Jackman RB. The use of nanodiamond monolayer coatings to promote the formation of functional neuronal networks. Biomaterials 2010;31:2097-104.

[3] Hees J, Kriele A, Williams OA. Electrostatic self-assembly of diamond nanoparticles. Chemical Physics Letters 2011;509:12-5.

[4] Zang J, Wang Y, Zhao S, Bian L, Lu J. Electrochemical properties of nanodiamond powder electrodes. Diamond and Related Materials 2007;16:16-20.

[5] Girard HA, Perruchas S, Gesset C, Chaigneau M, Vieille L, Arnault J-C, Bergonzo P, Boilot J-P, Gacoin T. Electrostatic Grafting of Diamond Nanoparticles: A Versatile Route to Nanocrystalline Diamond Thin Films. Acs Appl Mater Inter 2009;1:2738-46.

[6] Bergonzo P, Bongrain A, Scorsone E, Bendali A, Rousseau L, Lissorgues G, Mailley P, Li Y, Kauffmann T, Goy F, Yvert B, Sahel JA, Picaud S. 3D shaped mechanically flexible diamond microelectrode arrays for eye implant applications: The MEDINAS project. Irbm 2011;32:91-4.

[7] Specht CG, Tigaret CM, Rast GF, Thalhammer A, Rudhard Y, Schoepfer R. Subcellular localisation of recombinant alpha- and 
gamma-synuclein. Mol. Cell. Neurosci. 2005;28:326-34.

[8] Pool M, Thiemann J, Bar-Or A, Fournier AE. NeuriteTracer: A novel ImageJ plugin for automated quantification of neurite outgrowth. Journal of Neuroscience Methods 2008;168:134-9.

[9] Girard HA, Petit T, Perruchas S, Gacoin T, Gesset C, Arnault JC, Bergonzo P. Surface properties of hydrogenated nanodiamonds: a chemical investigation. Phys Chem Chem Phys 2011.

[10] Williams OA. Nanocrystalline diamond. Diamond and Related Materials 2011;20:621-40.

[11] Williams OA, Kriele A, Hees J, Wolfer M, Müller-Sebert W, Nebel CE. High Young's modulus in ultra thin nanocrystalline diamond. Chemical Physics Letters 2010;495:84-9.

[12] Sun KW, Wang JY, Ko TY. Raman spectroscopy of single nanodiamond: Phonon-confinement effects. Applied Physics Letters 2008;92:153115.

[13] Rainaldi G, Calcabrini A, Santini M. Positively charged polymer polylysine-induced cell adhesion molecule redistribution in K562 cells. J Mater Sci-Mater M 1998;9:755-60.

[14] Bacakova L, Filova E, Rypacek F, Svorcik V, Stary V. Cell adhesion on artificial materials for tissue engineering. Physiol Res 2004;53:S35-S45.

[15] Kotov N, (null). Inorganic Nanoparticles as Protein Mimics. Science 2010;330:188-9.

[16] Rao SS, Winter JO. Adhesion molecule-modified biomaterials for neural tissue engineering. Frontiers in Neuroengineering 2009;2:1-14.

[17] García A. Get a grip: integrins in cell-biomaterial interactions. Biomaterials 2005;26:7525-9.

[18] Shimaoka M, Springer T. Therapeutic antagonists and conformational regulation of integrin function. Nat Rev Drug Discov 2003;2:703-16.

[19] Mager MD, LaPointe V, Stevens MM. Exploring and exploiting chemistry at the cell surface. Nat Chem 2011;3:582-9.

[20] Hynes RO. Integrins: Bidirectional, Allosteric Signaling Machines. Cell 2002;110:673-87.

[21] Steele J, Dalton B, Johnson G, Underwood P. Polystyrene Chemistry Affects Vitronectin Activity - an Explanation for Cell Attachment to Tissue-Culture Polystyrene but Not to Unmodified Polystyrene. Journal of Biomedical Materials Research 1993;27:927-40.

[22] Steele J, Dalton B, Johnson G, Underwood P. Adsorption of Fibronectin and Vitronectin Onto Primaria(Tm) and TissueCulture Polystyrene and Relationship to the Mechanism of Initial Attachment of Human Vein Endothelial-Cells and Bhk-21 Fibroblasts. Biomaterials 1995;16:1057-67.

[23] Wasdo SC, Barber DS, Denslow ND, Powers KW, Palazuelos M, Stevens SMJ, Moudgil BM, Roberts SM. Differential binding of serum proteins to nanoparticles. Int J Nanotechnol 2008;5:92-115. 
[24] Wang H-D, Niu CH, Yang Q, Badea I. Study on protein conformation and adsorption behaviors in nanodiamond particleprotein complexes. Nanotechnology 2011;22:145703.

[25] Roach P, Farrar D, Perry CC. Surface tailoring for controlled protein adsorption: Effect of topography at the nanometer scale and chemistry. J Am Chem Soc 2006;128:3939-45.

[26] Roach P, Eglin D, Rohde K, Perry CC. Modern biomaterials: a review-bulk properties and implications of surface modifications. J Mater Sci: Mater Med 2007;18:1263-77. 
Figures

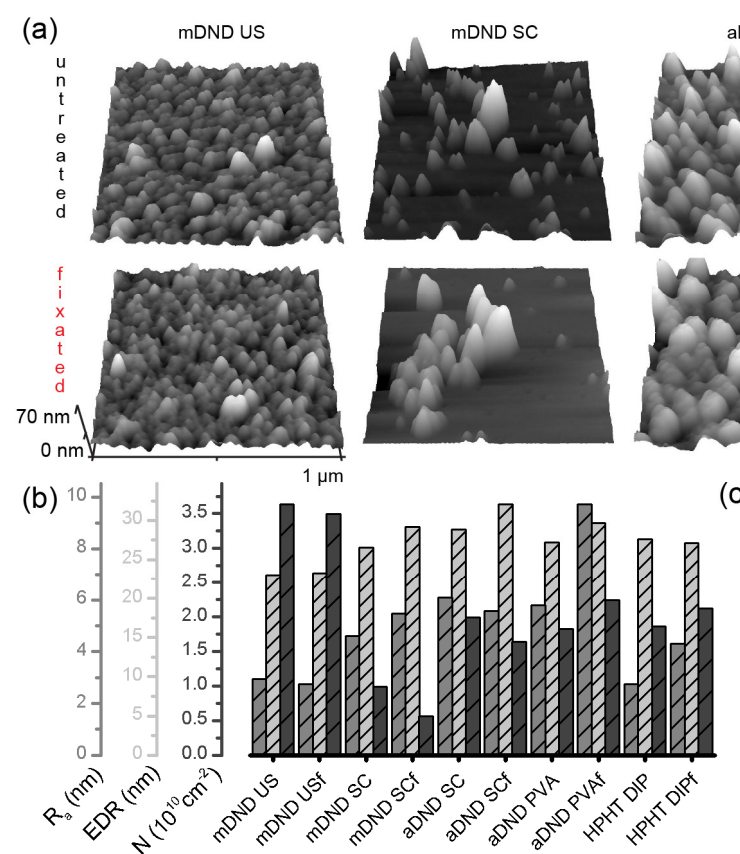

aDND SC
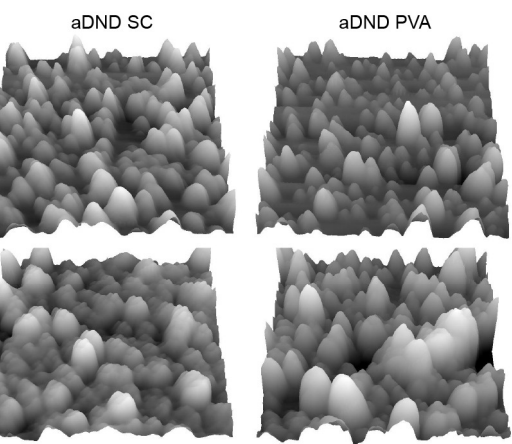

(c) $280-1 \quad$ 二

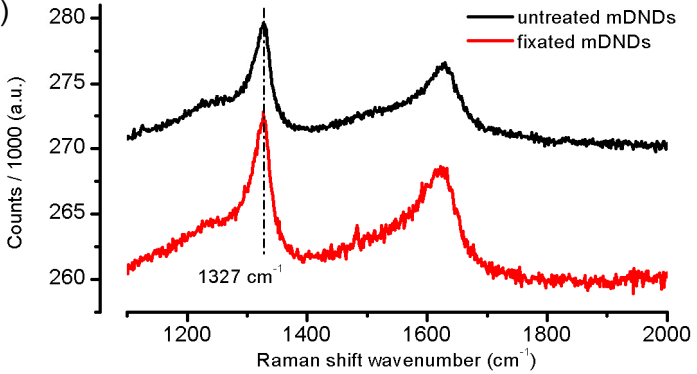

Figure 1: (a) $1 \mu \mathrm{m}$ square AFM images of ND-coated substrates coated using a variety of ND types and deposition methods. Row 1 are untreated coatings and Row 2 are MWPECVD fixated resulting in a hydrogenated surface. No differences in coating are apparent before and after fixing. Scans are representative of the whole sample surface and were selected at random over the entire substrate surface. (b) Corresponding average roughness, $\mathrm{R}_{\mathrm{a}}$; equivalent disc radius, EDR; and seeding density, $\mathrm{N}$ of AFM images. EDR measurements are subject to AFM tip convolution contributions resulting in an overestimate of particle size by ca. $10 \mathrm{~nm}$. (c) Raman spectra of untreated and fixated mDNDs show no significant differences in spectra. Pertinently the $1327 \mathrm{~cm}^{-1}$ peak has not shifted, indicating no change in core particulate size from the fixing treatment. 

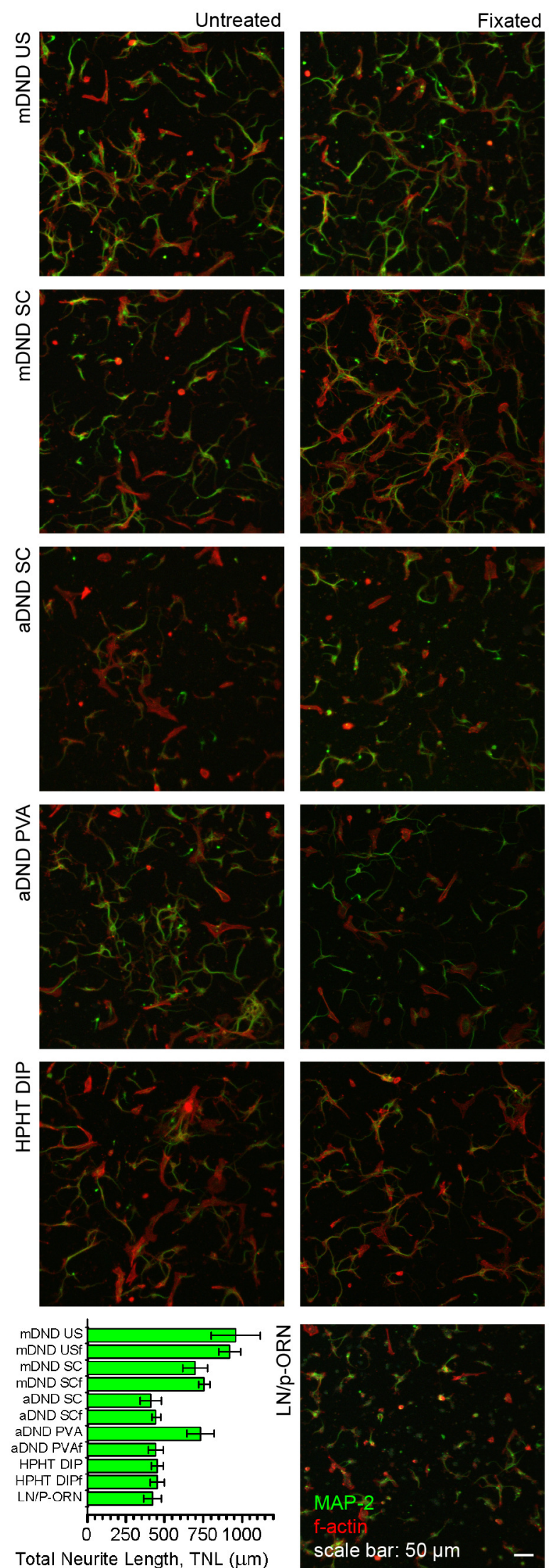

Figure 2: Confocal microscopy images of immunostained neuronal cell cultures after DIV2 on ND-coated and laminin (LN)/poly-DL-ornithine ( $\mathrm{p}^{-}$ 
ORN) coated glass substrates. Immunostaining reveals neurons via the dendrite-specific marker MAP2 (shown in green) and cytoskeletal filaments of actin (f-actin) are stained for using rhodamine-phalloidin (red), which highlights structures rich in f-actin, such as growth cones at the tips of neurites. All ND-coated samples promote neuronal adhesion and neurite outgrowth in a similar or favourable comparison to protein coated substrates. Notably, hydrogenated and untreated samples show very similar levels of attachment and outgrowth. Scale bar $50 \mu \mathrm{m}$. The quadrant-averaged Total Neurite Length (TNL) of each sample (measured using the NeuriteTracer ImageJ plugin) is shown in the bottom left panel, with error bars corresponding to the standard error of the mean (s.e.m.).

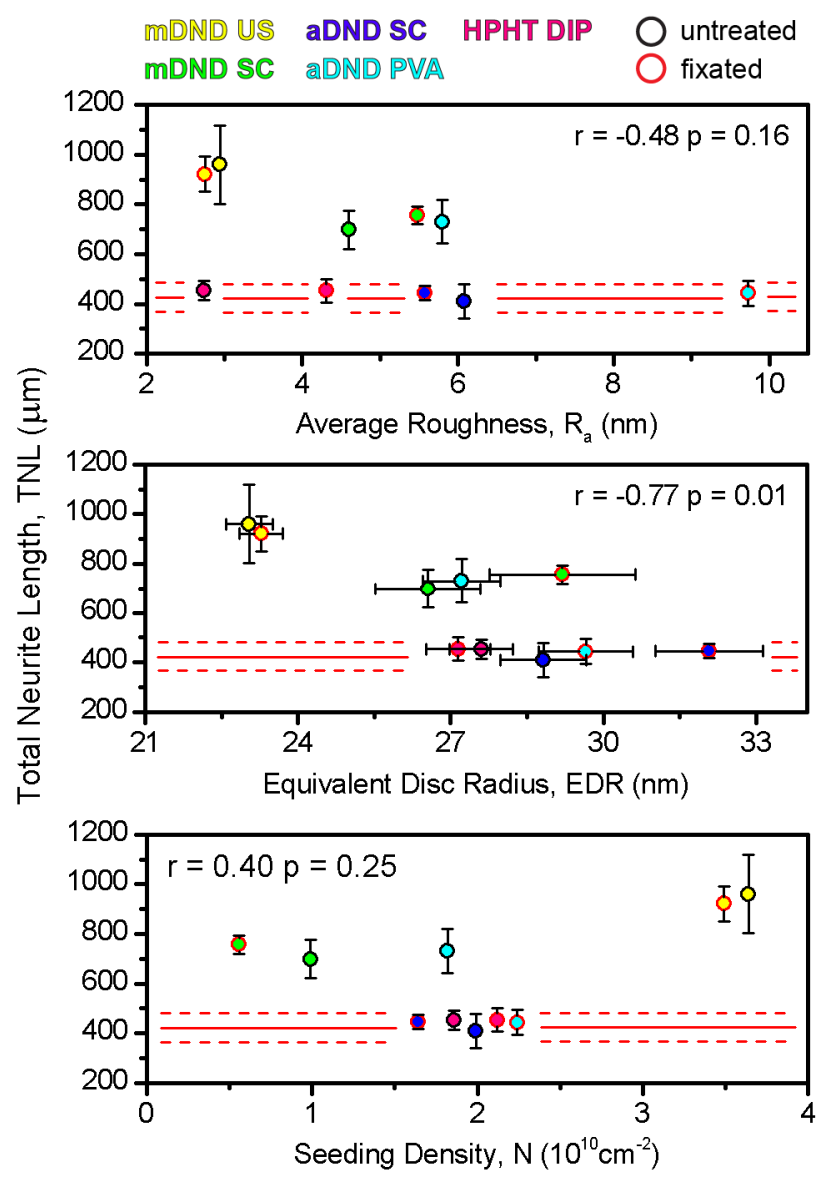

Figure 3: Scatter plots showing the relationships between the AFMdetermined morphological properties (Figure 1b) of the ND-coated samples against the total neurite extension measured from confocal microscopy images shown in Figure 2. Data point fill colour: ND coating type. Line 
border colour: untreated/fixated. TNL and EDR error bars are both s.e.m.. The solid horizontal line across each panel indicates the LN/p-ORN TNL with the flanking dashed lines indicating \pm s.e.m.. Inset $r$ and $p$-values report the Pearson correlation coefficient and 2-tailed significance for each data set.

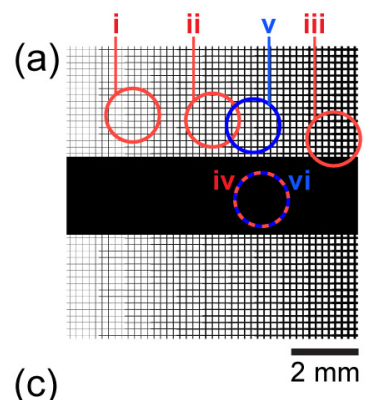

\section{DIV2}
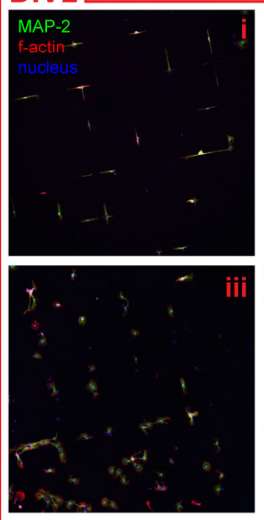
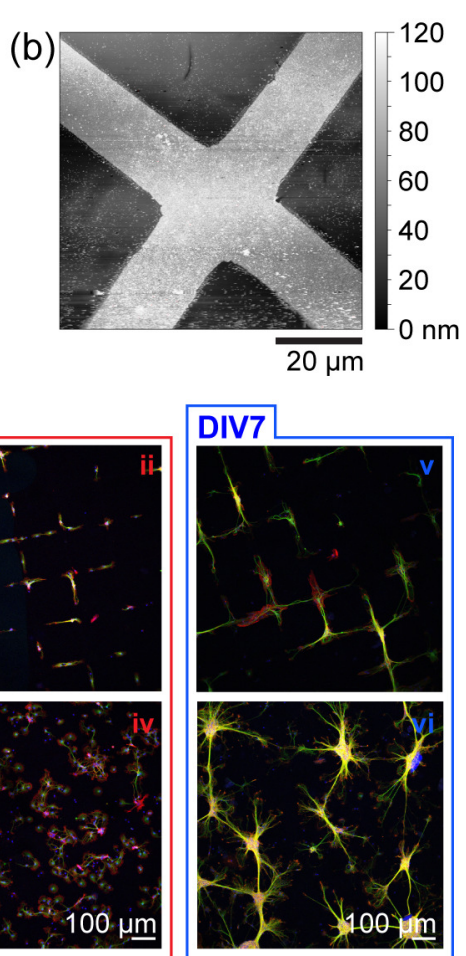

Figure 4: (a) Diagram of photolithography mask used for ND patterning (9 $\mathrm{mm}$ square). Black areas are ND filled. Grid pitch is $200 \mu \mathrm{m}$ and incremental gradated line widths of 5, 10, 20, 25, 30, 35, 40, 50, 60 and 70 $\mu \mathrm{m}$ increase from left to right every $4^{\text {th }}$ to $5^{\text {th }}$ grid unit. The central unpatterned region is used as an ND control surface for each sample. The indicated circular regions correspond to the confocal images presented in panel (c). (b) $70 \mu \mathrm{m}$ square AFM image of patterned aDND SC tracks on glass substrate. Width: $15 \mu \mathrm{m}$. Height: ca. $35 \mathrm{~nm}$. (c) Confocal microscopy images of immunostained neuronal cultures on untreated aDND SC patterned glass substrates. Cells have MAP2 and f-actin staining as in Figure 2 and an additional nuclei stain using Hoechst 33258. After DIV2 neurons can be seen to be following ND-tracks down to at least $10 \mu \mathrm{m}$ (c.i) and for larger line widths as well (c.ii, c.iii). After DIV7, neurons have 
formed interconnected networks as directed by the ND patterning (c.v). Scale bar $100 \mu \mathrm{m}$. 